\title{
Senegal: Explorer le processus de la collaboration multisectorielle mise en place pour l'étude qualitative
}

\author{
Fatou Mbow \\ Population Council \\ Nafissatou Diop \\ Awa Diop Dabo \\ Awa Tounkara Cissé \\ Seynabou Ba Diakhaté
}

Follow this and additional works at: https://knowledgecommons.popcouncil.org/departments_sbsr-rh

Part of the Demography, Population, and Ecology Commons, Family, Life Course, and Society Commons, International Public Health Commons, Maternal and Child Health Commons, Obstetrics and Gynecology Commons, and the Women's Health Commons How does access to this work benefit you? Let us know!

\section{Recommended Citation}

Mbow, Fatou, Nafissatou Diop, Awa Diop Dabo, Awa Tounkara Cissé, and Seynabou Ba Diakhaté. 2017. "Senegal: Explorer le processus de la collaboration multisectorielle mise en place pour l'étude qualitative," STEP UP Case Study. Dakar: Population Council. 


\section{SENEGAL: Explorer le processus de la collaboration multisectorielle mise en place pour l'étude qualitative}

LE PREUVE ET L'IMPACT: Examiner comment les activités de STEP UP ont éclairé les processus ou stratégies efficaces

Le but de la recherche opérationnelle sur la planification familiale et la santé reproductive est de recueillir des données ou des processus qui assistent les politiques et les programmes à maximiser l'accès des femmes aux services de qualités. Mais l'étape essentielle est d'assurer que ces données ou processus sont utilisées, et cette étape reçoit souvent l'attention insuffisante. De plus, il y a relativement peu de reportage et d'évaluation quant au fait de savoir si des résultats sont utilisées ou non.

Le but de cette étude de cas est de documenter une stratégie ou un processus d'une activité du consortium des programmes de recherche STEP UP qui a été efficace. Elle permet d'explorer les caractéristiques de l'activité qui l'ont rendue efficace, discuter les leçons apprises, et démontrer l'impact positif du STEP UP sur les programmes, politiques, ou communauté de recherche sur la planification familiale et la santé reproductive.

\section{RESUME}

En 2015, STEP UP a mené une étude qualitative sur l'expérience de femmes ayant subi une grossesse non désirée suite à un viol ou inceste dans la région de Dakar, en partenariat avec le Comité de plaidoyer pour l'accès à l'avortement médicalisé, plus connu sous le nom de « Taskforce ». L'objectif général de l'étude était de développer une meilleure compréhension de la vie de ces femmes et d'apporter leur voix au débat sur la légalisation de l'avortement médicalisé.

Pour la réalisation des objectifs spécifiques de l'étude, le Population Council (membre de STEP UP) a collaboré avec trois Organisations de la Société Civile (OSC) membres de la Taskforce : le Réseau Siggil Jigeen (RSJ) ; l'Association des Juristes Sénégalaises (AJS); et l'Association des Femmes Médecins du Sénégal (AFEMS).

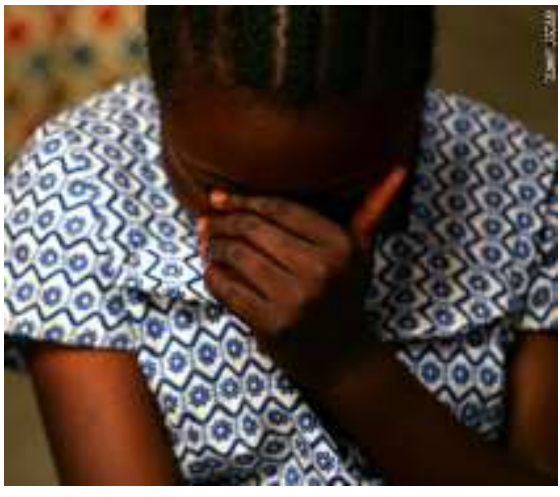

Doublement victime!

La collaboration avec la Taskforce, en général, et les trois OSC, en particulier, a été effective depuis la conception de l'étude. Cette étude de cas examine les caractéristiques de la collaboration, comment elle a été efficace, les leçons apprises, et les recommandations formulées pour de telles futures collaborations. 


\section{INTRODUCTION}

Au Sénégal, en 2010, environ 3.6 pour cent des décès maternels ont été liés à un avortement à risque ${ }^{1}$ et 51 500 avortements provoqués ont été reportés en 20121 . Pourtant, le Sénégal a ratifié des traités et conventions relatifs à la santé de la femme, au niveau régional et international. Malgré cela, le pays a l'une des lois les plus restrictives du monde sur l'avortement.

En 2013, le Comité de plaidoyer pour l'accès à l'avortement médicalisé au Sénégal (aussi appelé " Taskforce ») a renforcé ses activités de plaidoyer pour un assouplissement des textes relatifs à l'avortement. Depuis, l'avortement est plus ouvertement discuté. Cependant, la voix des femmes victimes de grossesse non désirée n'est pas entendue.

\section{LE PROJET}

En 2015, dans le cadre du Projet STEP UP, le Population Council a mené une étude qualitative sur l'expérience de femmes ayant subi une grossesse non désirée dans la région de Dakar, en partenariat avec la Taskforce. L'objectif général de cette étude était de développer une meilleure compréhension de la vie des femmes qui ont subi une grossesse non désirée suite à un viol ou un inceste, et d'apporter leur voix au débat sur la légalisation de l'avortement médicalisé. Les objectifs spécifiques étaient de :

- documenter la vie des femmes qui ont subi une grossesse non désirée résultant d'un viol ou d'un inceste et qui n'ont pas interrompu la grossesse (Groupe 1);

- documenter la vie des femmes qui ont été reconnues coupables d'avortement illégal ou d'infanticide et qui ont été sanctionnées par la loi (Groupe 2) ;

- documenter la vie des femmes dont l'état de santé s'est dramatiquement dégradé en raison d'une grossesse suite à un viol ou un inceste (Groupe 3); et

- fournir des évidences basées sur les recherches à la Taskforce et à tous ceux qui sont intéressés par le sujet pour servir dans les activités de plaidoyer pour un assouplissement des textes sur l'avortement.

Pour la réalisation de ces objectifs spécifiques, le Population Council a collaboré avec les trois (3) Organisations de la Société Civile (OSC) suivantes membres de la Taskforce: 1) Le Réseau Siggil Jigeen (RSJ) pour mener les Entretiens Approfondis (EA) avec des victimes du Groupe 1 ; 2) l'Association des Juristes Sénégalaises (AJS) pour le Groupe 2 ; et l'Association des Femmes Médecins du Sénégal (AFEMS) pour le Groupe 3.

\section{IMPACT DE LA COLLABORATION}

\section{Utilisation des résultats de la recherche}

Les publications de l'étude seront largement partagées aussi bien au niveau national qu'international.

En tant qu'utilisateur principal des résultats de l'étude, la Taskforce se les est appropriés et s'en sert déjà comme élément d'information et outil de plaidoyer auprès des parlementaires et acteurs clés (Ministères de la santé, de la famille, de la justice, etc.).

De plus, à l'occasion d'un atelier résidentiel tenu en maijuin 2016, les partenaires de l'étude ont développé un plan de dissémination et d'utilisation des évidences où chaque partie prenante a détaillé une série d'actions concrètes à mener dans les mois suivants.

\section{Publications sur les résultats de l'étude}

A partir des rapports de terrain et de l'analyse des verbatim, le Population Council a élaboré un premier draft de rapport de recherche qui a été finalisé avec les OSC lors d'une retraite organisée du 31 mai au 4 juin 2016.

Plusieurs présentations ont été faites pour partager largement les résultats de l'étude :

- Présentation à la $5^{\text {ème }}$ rencontre internationale de recherche sur les grossesses non désirées et l'avortement à risque à Mexico City, le 28 septembre 2015. Titre de la présentation « I was raped and got pregnant. Abortion is illegal in Senegal. What do I do?».

- Présentation des résultats à la Taskforce le 04 novembre 2015 pour prendre en compte leur feedback dans le rapport.

- Rencontre de partage auprès des parties prenantes au niveau national (20 Janvier 2016).

- Présentation aux parlementaires et acteurs clés lors de la réunion de la taskforce (le 19 mai 2016).

- Présentation au personnel des Représentants au Congrès américain pour un plaidoyer sur l'importance du financement des activités touchant à l'avortement dans les pays en développement (30 juin 2016).

La présente étude de cas a été développée pour partager l'expérience des partenaires de l'étude qui a été une collaboration extraordinaire pour chacun des collaborateurs. 


\section{LE PROCESSUS DE}

\section{DEVELOPPEMENT D'UN MODELE EFFICACE DE COLLABORATION}

\section{Cadre de la collaboration}

L'idée de mener une telle étude a été partagée dès le début par le Population Council avec toute la Taskforce. Les OSC retenues ont été choisies parce qu'elles ont I'habitude de travailler avec les différents profils/groupes de femmes victimes ciblées. En effet, afin de protéger l'anonymat des femmes et pour qu'elles se sentent plus à l'aise à participer à l'étude, seules les OSC avaient un accès direct aux victimes de leur groupe. Le Population Council qui n'avait pas de contact direct avec les interviewées, a toutefois veillé étroitement au respect des normes d'éthique de recherche et à la qualité technique de l'étude.

La collaboration avec RSJ, AJS et AFEMS a été effective depuis l'écriture du protocole de recherche pour assurer la pertinence et la faisabilité des actions proposées. Elle s'est poursuivie pendant la mise en œuvre (formation, collecte des données, transcription des interviews), l'élaboration de publications et la dissémination des résultats. Cette collaboration a été matérialisée par la signature d'un mémorandum d'accord, avec un plan de travail et un budget spécifiques à chaque OSC.

Les équipes des OSC étaient composées d'une (1) coordonnatrice de l'étude et de trois (3) intervieweuses.

\section{Formation}

Les équipes ont joué un rôle actif dans l'organisation logistique des formations et l'identification des personnes à interviewer pendant les prétests. La formation a été menée au Population Council dans certains cas, et dans les locaux de l'OSC dans d'autres.

Au départ, chaque coordonnatrice devait proposer six (6) membres de son organisation. La coordonnatrice et ces six (6) personnes ont été formées durant trois (3) jours en recherche qualitative en sciences sociales, en général, et à la compréhension et collecte de données pour l'étude, en particulier. La formation a porté sur la conduite d'entretiens éthiques et scientifiquement valides, y compris des sujets tels que l'importance du consentement éclairé et comment l'obtenir; les techniques de conduite d'entretiens approfondis; la maîtrise du guide d'entretien; comment présenter un entretien transcrit ; la protection des données collectées; etc. Chaque OSC a eu à faire le prétest de ses outils de collecte avec au moins 1 victime. Après la formation, le choix final de trois (3) intervieweuses par OSC a été fait par le Population Council et l'OSC.

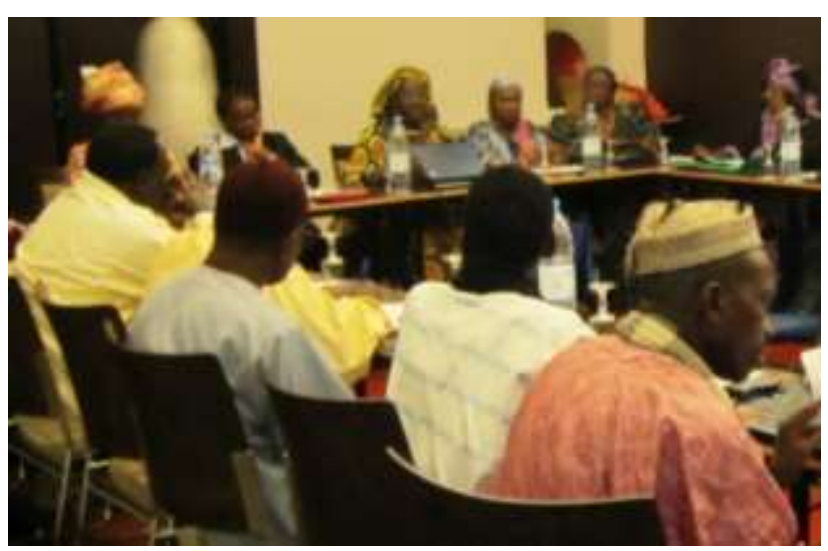

Le Taskforce a organisé un atelier avec des représentatives des familles religieuses les plus influents (29 August, 2013).

\section{Déroulement sur le terrain}

La collecte des données s'est déroulée entre Juin et Août 2015.

\section{Recherche de cas}

Pour le Groupe 1, RSJ a fait appel à ses Animatrices Communautaires (AC). II a aussi mis à contribution des structures telles que la Maison Rose, le Centre Guindi et le CEGID, qui s'activent dans l'encadrement et la prise en charge des victimes de violences basées sur le genre.

Pour le Groupe 2, les femmes éligibles ont été identifiées et abordées de 2 manières : 1) Pour les femmes détenues : avec l'assistance de la Direction de la Maison d'Arrêt pour Femmes (MAF) de Liberté VI ; et 2) Pour les femmes sorties de prison : avec l'assistance des membres de l'AJS et de leurs partenaires, ou d'anciennes détenues.

Pour le Groupe 3, l'AFEMS a informé ses membres de l'étude. Elle a également sollicité l'assistance de confrères appartenant aux associations d'obstétriciens/ gynécologues, de sages-femmes, ou des collaborateurs de la Maison Rose et du CEGID.

Certains cas, dont les critères de sélection ne correspondaient pas au groupe qui les ont identifiés, ont été référés au groupe intéressé.

\section{Interviews}

En respect des normes éthiques de recherche, chaque victime a commencé par signer un formulaire de consentement éclairé pour signifier sa participation volontaire. Les interviews ont duré en moyenne une (1) heure et se sont déroulées en général en une (1) ou deux (2) sessions. 


\section{Transcriptions}

Les transcriptions ont été effectuées par les intervieweuses immédiatement après les interviews. Le Population Council a ensuite réécouté chaque interview pour vérifier la précision de la transcription et mieux s'imprégner des discussions menées.

\section{Supervision}

Le Population Council a mené une supervision rapprochée des intervieweuses pour assurer la qualité des entretiens et de leurs transcriptions. Des rencontres régulières ont aussi été organisées avec les équipes des OSC pour le suivi de la mise en œuvre, et les informations utiles apprises d'un groupe ont été partagées avec les autres.

\section{Rapports de terrain}

Chaque OSC a fourni un rapport de terrain sur la base d'un canevas standardisé. Le rapport a résumé le bilan de la collecte, le déroulement des interviews et les difficultés rencontrées.

\section{Difficultés rencontrées}

\section{Par les OCS}

Plusieurs difficultés ont été communes à tous les groupes :

- L'identification des victimes suivant les critères d'éligibilité que les OSC ont trouvés trop rigoureux, notamment le fait que la grossesse soit issue d'un viol ou d'un inceste, que l'interviewée doive avoir au moins 18 ans au moment de l'interview, et que l'étude soit limitée à la région de Dakar.

- Le refus de cas potentiels de participer à l'étude car ne voulant plus avoir à se remémorer ou à parler d'épisodes douloureux de leur vie.

- L'identification du nombre prévue de cas pendant la période de collecte dont la durée ne pouvait être rallongée.

- Les difficultés liées à la disponibilité des équipes des OSC du fait de leurs autres occupations.

Chaque OSC a aussi fait face à des difficultés spécifiques, notamment :

- Groupe 1 : le fait que l'enfant issu du viol et de l'inceste devait avoir survécu au moins un (1) an. En effet, bien qu'il y ait une explication technique justifiant l'âge minimum d'un an pour l'enfant au moment de l'étude, il s'est trouvé que plusieurs femmes identifiées sur le terrain avaient des enfants de moins d'un (1) an. Cela faisait d'elles des cas inéligibles. En fait, le critère de l'âge de l'enfant a été le plus contraignant dans l'identification de cas éligibles.

- Groupe 2: l'impossibilité de mener l'interview lorsque le personnel de la MAF n'était pas disponible pour permettre l'accès à une détenue ; La difficulté de trouver des cas d'avortements illégaux car ceux de la MAF qui étaient potentiellement éligibles venaient juste d'être transférés hors de la zone de l'étude ; la lourdeur des procédures rencontrée dans certaines administrations pénitentiaires ; La difficulté de qualifier effectivement de viol ou d'inceste les cas potentiels à partir des déclarations ambiguës des détenues ellesmêmes; Le fait que, malgré l'explication initiale de l'objectif de l'étude, certaines détenues ont nourri l'espoir que leur participation pourrait contribuer à une meilleure prise en charge de leur dossier. Ces femmes se sont ensuite rétractées lorsqu'elles ont compris que l'étude ne les aiderait pas avec leur dossier ; Le refus des ex-détenues de participer à l'étude par manque de confiance suite à un antécédent avec les médias où, au cours d'une interview dans le cadre d'une activité avec d'autres acteurs, des victimes qui tenaient à leur anonymat ont quand même pu être identifiées.

- Groupe 3 : le fait que la complication grave de santé devait être liée à une grossesse issue de viol ou d'inceste ; La difficulté de trouver des familles de victimes décédées suite à une grossesse due à un viol ou un inceste.

\section{Par le Population Council}

- Le manque d'accès direct aux victimes par le Population Council. Les entretiens ont été menés par des intervieweuses qui n'étaient pas des spécialistes en recherche (notamment en recherche qualitative), même si certaines avaient une expérience en matière d'enquêtes. Aussi, il a fallu mettre un accent particulier sur la formation des OSC, sur le suivi de la collecte pour le respect des procédures éthiques et scientifiques, et sur l'importance de la qualité des données.

- Le retard dans la finalisation des transcriptions par certaines intervieweuses, ce qui a retardé le commencement de l'analyse. 


\section{LECONS APPRISES}

\section{Spécifiques aux OSC}

Les OCS ont rapporté les leçons suivantes qu'elles tirent de la conduite de cette étude :

\section{Avantages :}

- La coordination des efforts permet une bonne collaboration entre les partenaires. Cela a permis par exemple à des OSC de transférer à d'autres des cas potentiellement pertinents mais qui ne répondaient pas à leurs critères d'éligibilité.

- L'utilisation des partenariats et réseaux existants est un atout pour l'identification de cas. Ainsi, pour le Groupe 2, le partenariat existant déjà entre l'AJS et la MAF de Liberté $\mathrm{VI}$ a beaucoup facilité la recherche de cas. Pour le Groupe 3, l'AFEMS a pu retrouver et interviewer des victimes éligibles pour son groupe grâce à son réseautage.

- La collaboration continue avec le Population Council a permis un bon suivi du déroulement technique de l'étude (réunions, briefings).

- Le partenariat avec le Population Council a permis à AFEMS de conduire une recherche qualitative en sciences sociales qui sort de son cadre habituel d'analyse quantitative d'informations sanitaires.

\section{Défis}

- Une pareille étude exige des capacités en recherche qualitative. L'expérience antérieure de conduite d'enquêtes (quantitatives) n'est pas une garantie pour la bonne conduite d'entretiens qualitatifs.

- Les explications données n'ont pas toujours été bien comprises par les détenues. Malgré les informations données, des confusions ont persisté pour certaines détenues entre les objectifs de la recherche et une potentielle assistance juridique et judiciaire.

- Même si les partenaires habituels et réseaux existants peuvent être un atout, leur concours n'est pas toujours évident et ils n'ont parfois pas pu apporter la contribution espérée.

\section{Spécifiques aux Population Council}

\section{Avantages}

- Dans un partenariat où l'institution de recherche n'a pas accès direct aux victimes, il est essentiel d'assurer une formation rigoureuse en recherche des partenaires de mise en œuvre ainsi qu'un suivi rapproché pour le respect des procédures éthique et scientifique.

\section{Défis}

- Le non-accès direct aux personnes interviewées peut être une source de manque d'opportunités de mieux exploiter les réponses des personnes interviewées en ne probant pas d'avantage des informations potentiellement riches.

- En respect des normes éthiques, et pour éviter un risque de violation de la confidentialité et de l'anonymat des victimes après la collecte des données, il est important de s'assurer que les partenaires de mise en œuvre suppriment effectivement toute trace des interviews à leur disposition.

\section{Applicables à tous les partenaires}

\section{Avantages}

- L'implication des OSC dès la conception de l'étude leur en facilite la mise en œuvre et l'appropriation. Cela permet aussi une meilleure prise en compte de la réalité des OSC sur le terrain, et peut faciliter l'utilisation des résultats de la recherche.

- Une pareille étude peut renforcer la synergie d'efforts entre les différents collaborateurs travaillant dans des domaines complémentaires.

\section{Défis}

- La non-disponibilité du personnel des OSC peut être un frein à une bonne mise en œuvre.

- $\quad$ ll est important de respecter les critères d'éligibilité d'une étude conduite de manière éthique et scientifique. Cependant, cela peut rendre plus compliqué l'identification des cibles, malgré les nombreux cas rapportés par la presse, les cas abondants d'abus sexuel suivis de grossesse reçus dans les boutiques de droits de l'AJS, et le nombre important de femmes détenues pour infanticide ou avortement souvent recensées lors des visites des femmes juristes dans les prisons. 


\section{Recommandations pour de telles futures collaborations}

\section{Pour la recherche}

- Explorer la possibilité d'assouplir les critères d'éligibilité pour la sélection des cas.

- Encourager la collaboration entre les OSC, qui peut permettre de faciliter la référence de cas d'un groupe à l'autre.

- Prévoir une souplesse du calendrier pour permettre d'atteindre l'échantillon ciblé.

- Prévoir une réplication de cette recherche sur l'étendue du territoire national.

- Penser à une étude prospective avec un questionnaire administré immédiatement après consultation pour soins après avortement afin d'avoir une meilleure compréhension des types d'avortement et du profil des victimes.

- S'assurer que les partenaires appliquent correctement les principes de recherche et d'éthique selon les termes du protocole de recherche.

- Exiger la disponibilité de tous les partenaires durant tout le processus pour le bon déroulement des activités.

\section{Pour le plaidoyer/accompagnement des victimes}

- Assurer une large utilisation des évidences de la recherche pour le plaidoyer pour l'accès à l'avortement médicalisé à travers divers canaux : Utilisation d'extraits de l'étude dans des productions vidéo ou flyers ; présentations lors d'ateliers, conférences, causeries, animations radio ou télé, etc.

- Faire le plaidoyer auprès des bailleurs de fond pour la prise en charge totale des victimes (numéro vert, aide psychologique et juridique, accompagnement économique pour la réhabilitation de leur dignité, etc.).

- Etudier la possibilité de mettre en place une base de données sécurisée pour répertorier les cas de viol ou d'inceste suivi de grossesse afin de faciliter leur accompagnement et les recherches scientifiques.

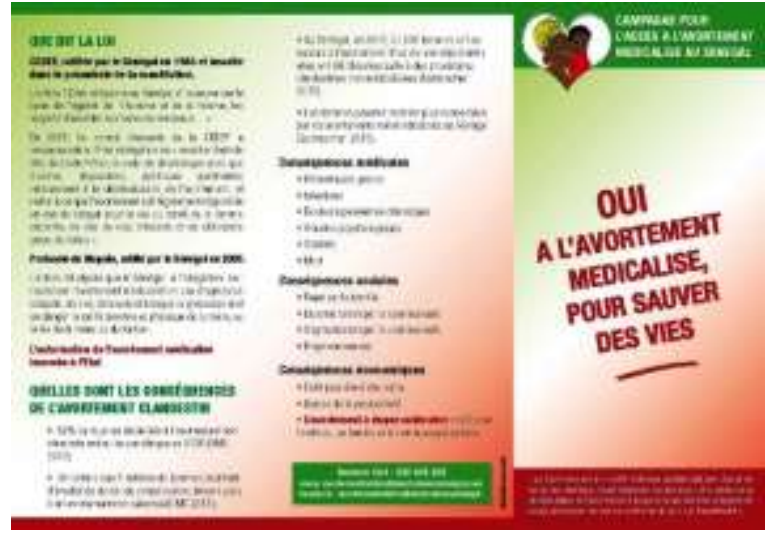

Matériel de campagne de la «Taskforce »

\section{CONCLUSION}

Le partenariat mis en place entre le Population Council et les OSC a permis de mener à bien la recherche proposée malgré les difficultés rencontrées. Toutefois, pour garantir des données de qualité qui respectent des principes éthiques et scientifiques, des mesures spécifiques s'avèrent nécessaires.

Les résultats issus de cette recherche pourront ainsi contribuer à des efforts de plaidoyer étayés par des données probantes, et à faire entendre la voix des victimes dans les débats juridiques, politiques, sociaux et religieux autour de la légalisation de l'avortement médicalisé au Sénégal.

\section{LECTURE SUGGEREE}

1. Fatou Bintou Mbow, Nafissatou Diop, Awa Diop Dabo, Awa Tounkara Cissé, Seynabou Ba Diakhaté. 2016. "L'expérience de femmes ayant subi une grossesse non désirée au Sénégal: Une étude qualitative " Rapport de recherche. STEP UP. Dakar: Population Council

\section{Citation recommandée :}

FB Mbow, N Diop, A Diop Dabo, A Tounkara Cissé, S Ba Diakhaté. 2017. "SENEGAL: Explorer le processus de la collaboration multisectorielle mise en place pour l'étude qualitative." Etude de cas STEP UP. Dakar: Population Council. 